\title{
Self-Calibration of Neutrino Detectors using characteristic Backgrounds
}

\author{
Joachim Kopp回 Manfred Lindner and Alexander Merl@ \\ Max-Planck-Institut für Kernphysik \\ Postfach 103980 \\ D-69029 Heidelberg \\ Germany
}

\begin{abstract}
We introduce the possibility to use characteristic natural neutrino backgrounds, such as Geoneutrinos $\left(\bar{\nu}_{e}\right)$ or solar neutrinos $\left(\nu_{e}\right)$, with known spectral shape for the energy calibration of future neutrino detectors, e.g. Large Liquid Scintillator Detectors. This "CalEffect" could be used without the need to apply any modifications to the experiment in all situations where one has a suitable background with sufficient statistics. After deriving the effect analytically using $\chi^{2}$ statistics, we show that it is only tiny for reactor neutrino experiments, but can be applicable in other situations. As an example, we present its impact on the identification of the wiggles in the power spectrum of supernova neutrinos caused by Earth matter effects. The Self-Calibration Effect could be used for cross checking other calibration methods and to resolve systematical effects in the primary neutrino interaction processes, in particular in the low energy cross sections.
\end{abstract}

\section{INTRODUCTION}

As neutrino physics is entering the stage of precision measurements, future detectors will have to face new challenges in terms of background reduction, control of systematical uncertainties, and calibration of the counting efficiencies and the energy reconstruction. The most widely used techniques for performing the energy calibration are radioactive sources with known properties, artificially accelerated lepton beams, or indirect methods based on the interaction products of neutrinos (see e.g. Refs. [1, 2, 3]). All of these methods have in common that they are only sensitive to charged particles, i.e. possible uncertainties associated with the primary neutrino interaction that produces these particles are not taken into account. Therefore it is desirable to calibrate the detector directly with a neutrino beam. Such a measurement has been performed by GALLEX using a Cr-51 neutrino source, but it was very involved and required a special detector design [4]. The SAGE experiment has also been calibrated with neutrinos, from Cr-51 [5] and Ar-37 [6].

In this letter, we propose to use natural neutrino sources with known characteristic spectra, in particular Geo-neutrinos and solar neutrinos as calibration sources for future low-energy neutrino detectors. These types of neutrinos are always present, and, in a several-tens-ofkilotons detector, they induce at least a few thousands of events per year. The energy spectra for both, Geoneutrinos $\left(\bar{\nu}_{e}\right)$ and solar neutrinos $\left(\nu_{e}\right)$, are known very precisely since their production processes are very well understood. They exhibit characteristic steps at the cutoff energies of various processes, which are easy to locate in the final event spectrum even if the total rate is not known.

\footnotetext{
a Email: Joachim.Kopp@mpi-hd.mpg.de

bEmail: Manfred.Lindner@mpi-hd.mpg.de

${ }^{c}$ Email: Alexander.Merle@mpi-hd.mpg.de
}

Of course, the energy calibration with Geo-neutrinos or solar neutrinos will not eliminate the need for other calibration methods, in particular because it is insensitive to spatial or temporal variations of the detector performance and can be used only in the very low energy region, but it is nevertheless interesting since it constitutes a self-calibration of the detector and provides an independent cross check of other methods without requiring any modifications of the detector design.

In Sec. 2, we present the small effect of the detector self-calibration on reactor neutrino experiments and explain the effect analytically. Then, in Sec. 3, we will show how the Self-Calibration Effect can improve the accuracy of supernova neutrino measurements, and we finally conclude in Sec. 4 .

\section{BACKGROUND NEUTRINOS AS CALIBRATION SOURCES}

A future Large Liquid Scintillator Detector (LLSD) will have very good statistics even for background neutrino sources such as distant nuclear reactors and Geoneutrinos. This opens the interesting possibility to use these neutrinos as calibration sources for the energy reconstruction, which leads to the seemingly paradoxial situation that a measurement with backgrounds can yield more precise results than a measurement without backgrounds.

Geo-neutrinos are particularly well suited for this selfcalibration because their spectrum, which is given by the uranium and thorium decay chains, has characteristic steps at very well known energies that can easily be located in the data samples (see Fig. 11). An analogous self-calibration for $\nu_{e}$ 's may be possible using solar neutrinos since the spectrum of Be- 8 and pep is also very characteristic.

Of course, background neutrinos are insensitive to short-term variations of the detector properties such as temperature fluctuations, so they will not eliminate the need for other calibration methods. But they are the

Typeset by REVTEX 
only tool to resolve systematical effects in the primary neutrino interaction process such as uncertainties in the low energy neutrino-nucleon cross sections.

In this chapter, we will first decribe in which analysis we have found the self-calibration effect and then show that it can be explained analytically using the $\chi^{2}$ approach.

\subsection{The tiny Self-Calibration Effect in Reactor Neutrino Experiments}

Already in [7] we have discussed the physics potential of reactor experiments with a LLSD such as the proposed $45 \mathrm{kt}$ LENA detector [8, 9]. Using the GLoBES software [10, 11], we have investigated the accuracy of such an experiment to measurements of the reactor angle $\theta_{13}$ as well as on the solar oscillation parameters, $\theta_{12}$ and $\Delta m_{21}^{2}$, using mobile nuclear reactors amongst others. As backgrounds, we have taken into account the 20 closest reactors to the possible LENA site Pyhäsalmi in Finland as well as Geo-neutrinos coming from uranium and from thorium (the Geo-neutrinos from potassium are not included in the analysis, since their energies are below the threshold for inverse beta decay, which is used as detection reaction for the reactor- $\bar{\nu}_{e}$ 's in a LLSD, see Fig. 11). For $\theta_{13}$, these backgrounds turn out not to be very important: the optimum baselines for such a measurement are very short $(\sim 1 \mathrm{~km})$, which leads, due to the large fiducial mass of the considered detector, to extremely high event rates even for a mobile reactor. Hence, for a measurement of the small reactor angle, the perturbation by the two different backgrounds is so small that it is not possible to exploit any information on the spectral shape of Geo-neutrinos.

However, for a measurement of $\theta_{12}$, the situation is different: considering the $S M A L L$ scenario (details on this analysis can be found in [7]), which corresponds to a mobile nuclear reactor with a thermal power of $0.5 \mathrm{GW}_{\mathrm{th}}$ and 2 years of data taking, one can see a small effect induced by the background self-calibration of the experiment, which will be pointed out here. For the inclusion of Geo-neutrinos in our analysis, we have considered three different situations:

- No Geo-neutrinos: In this case, Geo-neutrinos are completely absent, and only the background from distant nuclear reactors is taken into account, which yields 850 events per year.

- Geo-neutrinos with a $\mathbf{1 0 \%}$ uncertainty: Here, Geo-neutrinos coming from uranium and from thorium are taken into account and the uncertainty in both their fluxes is assumed to be $10 \%$. The Geo-neutrino spectra in our simulations are taken from [12, 13]. The reactor background is the same as in the scenario without Geo-neutrinos.

- Geo-neutrinos with a $\mathbf{1 0 0 \%}$ uncertainty: This scenario is equivalent to the previous one, but now the uncertainties in the two Geo-neutrino contributions are taken to be $100 \%$.
The result of our analysis for the $S M A L L$ scenario is again plotted here in Fig. 2 (cf. Fig. 3 in [7]). The interesting region is the marked rectangle. Taking a closer look it turns out to show a seemingly paradoxial situation: from Fig. 3 one can see that, for certain baselines, the lower bound on the assumed true value of $\sin ^{2} 2 \theta_{12}=0.83$ is slightly better with the background by Geo-neutrinos (and even in both cases, with $10 \%$ and $100 \%$ flux uncertainty) than without. The reason is that this background has such a characteristic form due to the well-known $Q$-values of the nuclear production reactions of the neutrinos that the positions of the steps in the spectrum can, in the minization of the $\chi^{2}$ function, be used to reduce a systematical bias in the energy calibration essentially to zero, which may not be possible without such a characteristic background. Hence it can indeed be the case, that, for certain configurations, a suitable background can improve, rather than worsen, the sensitivity of certain kinds of experiments.

The effect for reactor neutrinos is very tiny. This is because one needs to have large background rates to be able to resolve the characteristic spectrum properly. Also the energy scale of the background is crucial since it may not be possible to extrapolate a calibration made by $\mathrm{MeV}$ neutrinos accurate enough e.g. to the GeV scale. However, the principle effect existis, as we will demonstrate in the next section (and what we have tested using different hypothetical benchmark scenarios), and one just has to find a scenario where this effect can be applied.

\subsection{Analytical discussion of the Self-Calibration Effect}

Let us now discuss the self-calibration effect analytically. We consider a simplified $\chi^{2}$ expression using a Gaussian approximation for the distribution of the event rates:

$$
\chi^{2}=\sum_{i}^{\# \text { bins }}\left[\frac{\left(T_{i}\left(a_{i}, b\right)-N_{i}\right)^{2}}{N_{i}}+\frac{a_{i}^{2}}{\sigma_{i}^{2}}\right] .
$$

Here, the $T_{i}$ 's are the theoretically predicted event rates calculated with certain test values for the neutrino oscillation parameters and the systematical errors, while the $N_{i}$ 's are the rates obtained by using the assumed "true" values for these parameters. The $a_{i}$ 's are nuisance parameters as e.g. overall normalization errors or bin-dependent shape errors. The explicit form of the $T_{i}$ 's is

$$
\begin{aligned}
T_{i} & =\left(1+a_{i}\right) \tilde{N}_{i}(b) \\
\tilde{N}_{i}(b) & =(1+b) \cdot\left[\left(N_{\lfloor\delta(i)\rfloor+1}-N_{\delta(i)}\right) \cdot\right. \\
& \left.\cdot(\delta(i)-\lfloor\delta(i)\rfloor)+N_{\lfloor\delta(i)\rfloor}\right] \\
\delta(i) & =b \cdot\left(i+t_{0}+\frac{1}{2}\right)+i .
\end{aligned}
$$

Here, $\tilde{N}_{i}(b)$ are the rates for wrong energy binning implied by a non-zero energy calibration $b$ which are obtained from the correctly binned rates $N_{i}$ according to 
Eq. (3), which is essentially a linear interpolation between the events in bin $\lfloor\delta(i)\rfloor+1$ and bin $\lfloor\delta(i)\rfloor . t_{0}$ is the energy threshold of the detector, expressed in terms of the bin width, and the Gauß bracket $|\cdot|$ denotes the floor function.

The normalization uncertainties are completely contained in the bin-to-bin normalization factors $a_{i}$ with errors $\sigma_{i}$ which contain all spectral distortion effects, such as imperfect knowledge of the reactor signal spectrum, neutrino oscillations, etc.. The energy calibration is parametrized by $b$. Normally, one would also have to include a penalty term for the energy calibration of the form $b^{2} / \sigma_{b}^{2}$ (as done for the spectral distortions). Such a term is omitted here because we assume no external information on $b$, which means that the energy calibration is initially completely arbitrary. We do, however, assume $b$ to be sufficiently small to take $\lfloor\delta\rfloor=i$ in Eq. (4) (this only means that wrongly binned events are not shifted by more than one energy bin) and to expand the whole $\chi^{2}$ function up to first order in $b^{2}, a_{i}^{2}$, and $b a_{i}$.

Combining the normalization and spectral uncertainties, $T_{i}$ is given by

$$
T_{i}=\left(1+a_{i}+b\right)\left[\left(N_{i+1}-N_{i}\right) \cdot b \cdot\left(i+t_{0}+\frac{1}{2}\right)+N_{i}\right] .
$$

Neglecting terms such as $\mathcal{O}\left(b^{3}\right)$ and higher in $\chi^{2}$ means neglecting terms of order $\mathcal{O}\left(b^{2}\right)$ or $\mathcal{O}\left(b a_{i}\right)$ in $T_{i}$. With this approximation, our $\chi^{2}$ function becomes

$$
\begin{aligned}
& \chi^{2}=\sum_{i}\left(\frac { 1 } { N _ { i } } \left[\left(1+a_{i}+b\right) N_{i}+b\left(N_{i+1}-N_{i}\right) .\right.\right. \\
&\left.\left.\cdot\left(i+t_{0}+\frac{1}{2}\right)-N_{i}\right]^{2}+\frac{a_{i}^{2}}{\sigma_{i}^{2}}\right) .
\end{aligned}
$$

This expression has to be minimized with respect to $b$ :

$$
\begin{aligned}
\frac{\partial \chi^{2}}{\partial b} & =\sum_{i} \frac{2}{N_{i}}\left[\left(a_{i}+b\right) N_{i}+b\left(N_{i+1}-N_{i}\right)\left(i+t_{0}+\frac{1}{2}\right)\right] . \\
\cdot & {\left[N_{i}+\left(N_{i+1}-N_{i}\right)\left(i+t_{0}+\frac{1}{2}\right)\right]=0 } \\
b & =-\frac{\sum_{i} \frac{1}{N_{i}} a_{i} N_{i} \gamma_{i}}{\sum_{i} \frac{1}{N_{i}} \gamma_{i}^{2}}
\end{aligned}
$$

where we have introduced the short hand notation

$$
\gamma_{i}=N_{i}+\left(N_{i+1}-N_{i}\right)\left(i+t_{0}+\frac{1}{2}\right) .
$$

There are two extreme cases: First, let us assume a very smooth energy spectrum, i.e. $N_{i+1}-N_{i} \ll N_{i}$. Then, $\gamma_{i} \approx N_{i}$, and it follows from Eq. (8) that $b$ is of the same order as $a_{i}$. However, if the energy spectrum contains several large steps (as the Geo-neutrino spectrum does), at least some of the differences $N_{i+1}-N_{i}$ are sizeable, so $\gamma_{i} \gg N_{i}$. As $\gamma_{i}$ enters the denominator of Eq. (8) quadratically, while the numerator exhibits only a linear dependence, the fit value of $b$ will be very small in this case (close to zero), i.e. the energy calibration uncertainty is essentially eliminated.

\section{APPLICATION TO EARTH MATTER EFFECTS ON SUPERNOVA NEUTRINOS}

On the one hand, the self-calibration effect is a nice tool to make an experimentalist's work more efficient. But to demonstrate that it can also help to do precision measurements, we consider the matter effects of the Earth on supernova neutrinos as an example using the same LLSD as in Sec. 2

The fact that Earth matter has an effect on the spectrum of supernova neutrinos has been presented in [14, 15]. A supernova core is essentially a neutrino blackbody source, so that the spectrum of $\bar{\nu}_{e}$ 's produced in the supernova is thermal. However, it is important to keep in mind that the neutrinos that are produced as $\bar{\nu}_{e}$ reach us as matter eigenstates $\bar{\nu}_{1}$, which means that the oscillations that appear inside the Earth are $\bar{\nu}_{1}-\bar{\nu}_{2}$ oscillations [16]. This has been taken into account in our simulations by modifying the source code of the GLoBES software accordingly.

The effect of the oscillations in the Earth on the neutrino energy spectrum is then, that one can see wiggles on the otherwise smooth spectrum. This can be shown more clearly by going at first to inverse energy units and then taking the squared modulus of the Fourier transform of the resulting spectrum, which gives the power spectrum, that points out the different oscillation modes. In the case of Earth matter effects, this results in one or more peaks, depending on whether the neutrinos traverse the core of the Earth or only the mantle. To extract these peaks from the experimental data, it is important to precisely know their positions. This obviously requires an excellent energy calibration 15].

As example for our calculations, we have taken the "accretion-phase model I" from [17. This gives the value $\alpha=4.4$ for the flux parameter as well as $\Phi\left(\bar{\nu}_{e}\right) / \Phi\left(\bar{\nu}_{x}\right)=$ 0.8 (for details, see [15]), where $x$ stands for all other neutrino flavours except $\bar{\nu}_{e}$. For modelling the Earth matter, we have taken two different scenarios: a constant density equal to the average mantle density of $4.6 \mathrm{~g} / \mathrm{cm}^{3}$ according to the PREM profile [18] as well as a 3 layer approximation with two times the mantle density, but a different core density of $11.8 \mathrm{~g} / \mathrm{cm}^{3}$. In both scenarios, the neutrinos travel through the whole Earth, which corresponds to a baseline of $12742 \mathrm{~km}$. Note that our normalization is made for approximately 2000 events in the detector, which is a good example value according to [14].

This has been simulated with the GLoBES software package for different cases (cf. Fig. 4). The first case is propagation in vacuum (black-dotted line). This does of course not have any effect on the power spectrum of neutrinos reaching the Earth from the supernova, simply because it is completey equivalent to a $12742 \mathrm{~km}$ longer propagation in space, which clearly makes no difference for a supernova whose expected distance from the Earth is several kpc. For propagation through matter, one can see - as expected - one or even more peaks, depending on the number of layers of constant density in the considered Earth model. The red-dashed curve with a wrong 
energy calibration ( $10 \%$ error) is clearly separated from the one with perfect calibration (green-solid). Of course, $10 \%$ is much worse than this error would be in reality, but here just the Self-Calibration Effect is to be demonstrated which is more illustrative for a larger error. The crosses are data points coming from a simulation that has also been started with an initial calibration error of $10 \%$, but in that case, we have fitted the energy calibration $b$ to the Geo-neutrino background. This can be done since the SN neutrinos can be easily separated from all other events simply because of the narrow time window for neutrinos coming from a supernova. This $\chi^{2}$ analysis then pulls the value of the energy calibration $b$ down to zero because of the self-calibration effect. Then, shifting the energy of the events by the best-fit value of the calibration gives no difference to the case of perfect energy calibration, which can be seen for both scenarios in Fig. 囵.

Note that in this calculation, we have assumed a perfect extrapolation of the energy calibration to higher energies. Geo-neutrinos have energies up to about $3.3 \mathrm{MeV}$ (cf. Fig. 11), while supernova neutrinos can have energies of several tens of MeV. Of course, in reality, this extrapolation cannot work perfectly, but may be possible to a good enough precision.

This shows that the Self-Calbration Effect can be a nice tool for measuring Earth matter effects on neutrinos produced in supernovae. However, this is just one example, but of course, the effect applies to all situations, where an accurate energy calibration is required and a background source with characteristic spectrum and good enough statistics is available. Possible applications of the solar neutrino spectrum should also be con- sidered.

\section{CONCLUSIONS}

We have presented the interesting possibility for a Large Liquid Scintillator Detector in future neutrino experiments to use natural backgrounds with a characteristic spectral shape for the energy calibration. Due to their well-known spectrum, this could be Geo-neutrinos as source of $\bar{\nu}_{e}$ 's or solar neutrinos as source of $\nu_{e}$ 's. We have demonstrated that this effect is only tiny for reactor neutrino experiments due to their high statistics and known spectral shape. However, we have also shown that the effect can be derived analytically and that it could be a nice tool in suitable scenarios, e.g. supernovae, where the correct energy calibration is crucial for detecting Earth matter effects on the neutrinos originating from such an explosion. The effect applies to all situations with a suitable background source and high enough statistics.

\section{Acknowledgments}

We would like to thank K. Hochmuth, W. Hampel, P. Huber, T. Marrodán-Undagoitia, M. Rolinec, M. Wurm, and especially L. Oberauer for useful discussions and information on the LENA detector as well as H.-T. Janka, G. Raffelt, and R. Tomàs for useful comments and information. We are grateful to S. Enomoto for sending us his data files on Geo-neutrino spectra in machine-readable form. This work has been supported by the Sonderforschungsbereich TR27 "Neutrinos and Beyond" der Deutschen Forschungsgemeinschaft.
[1] G. Alimonti et al. (Borexino), Astropart. Phys. 16, 205 (2002), hep-ex/0012030.

[2] K. Eguchi et al. (KamLAND), Phys. Rev. Lett. 90, 021802 (2003), hep-ex/0212021.

[3] M. Nakahata et al. (Super-Kamiokande), Nucl. Instrum. Meth. A421, 113 (1999), hep-ex/9807027.

[4] W. Hampel et al. (GALLEX), Phys. Lett. B420, 114 (1998).

[5] J. N. Abdurashitov et al. (SAGE), Phys. Rev. C59, 2246 (1999), hep-ph/9803418.

[6] J. N. Abdurashitov et al., Phys. Rev. C73, 045805 (2006), nucl-ex/0512041.

[7] J. F. Kopp, M. Lindner, A. Merle, and M. Rolinec, JHEP 01, 053 (2007), hep-ph/0606151.

[8] T. Marrodan-Undagoitia et al., Phys. Rev. D72, 075014 (2005), hep-ph/0511230.

[9] L. Oberauer, F. von Feilitzsch, and W. Potzel, Nucl. Phys. Proc. Suppl. 138, 108 (2005).

[10] P. Huber, M. Lindner, and W. Winter, Comput. Phys. Commun. 167, 195 (2005), hep-ph/0407333.

[11] P. Huber, J. Kopp, M. Lindner, M. Rolinec, and W. Winter (2007), hep-ph/0701187.

[12] S. Enomoto, Ph.D. thesis (2005).
[13] S. Enomoto, URL http://www.awa.tohoku.ac.jp/ sanshiro/geoneutrino/spectrum/.

[14] A. S. Dighe, M. T. Keil, and G. G. Raffelt, JCAP 0306, 006 (2003), hep-ph/0304150.

[15] A. S. Dighe, M. Kachelriess, G. G. Raffelt, and R. Tomas, JCAP 0401, 004 (2004), hep-ph/0311172.

[16] A. S. Dighe and A. Y. Smirnov, Phys. Rev. D62, 033007 (2000), hep-ph/9907423.

[17] M. T. Keil, G. G. Raffelt, and H.-T. Janka, Astrophys. J. 590, 971 (2003), astro-ph/0208035.

[18] A. M. Dziewonski and D. L. Anderson, Phys. Earth Planet. Interiors 25, 297 (1981). 


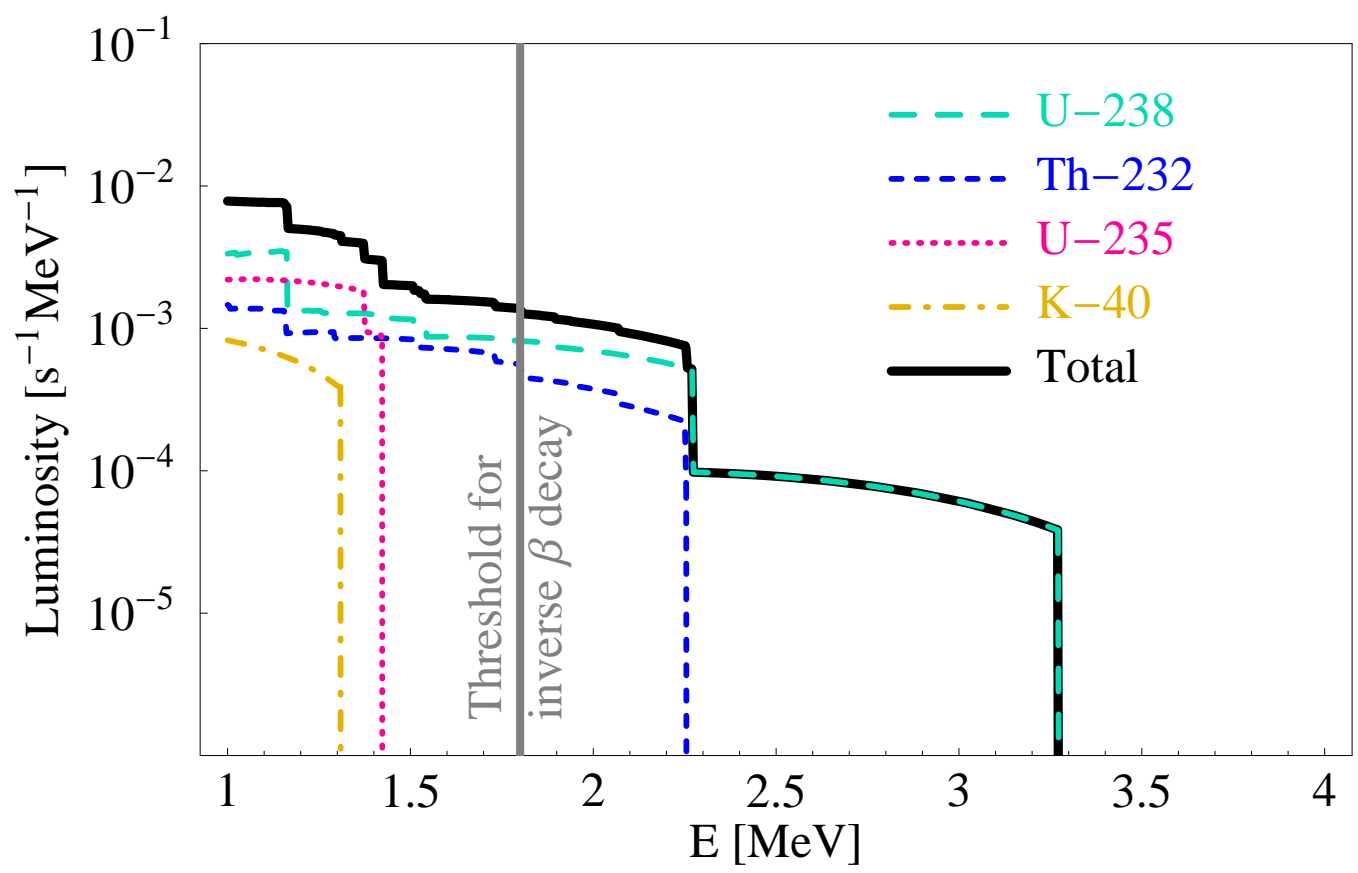

Figure 1: The energy spectrum of Geo-neutrinos [12, 13]. Note that only the decay chains of uranium-238 and thorium-232 produce neutrinos that are above the threshold of inverse $\beta$ decay at $1.8 \mathrm{MeV}$.

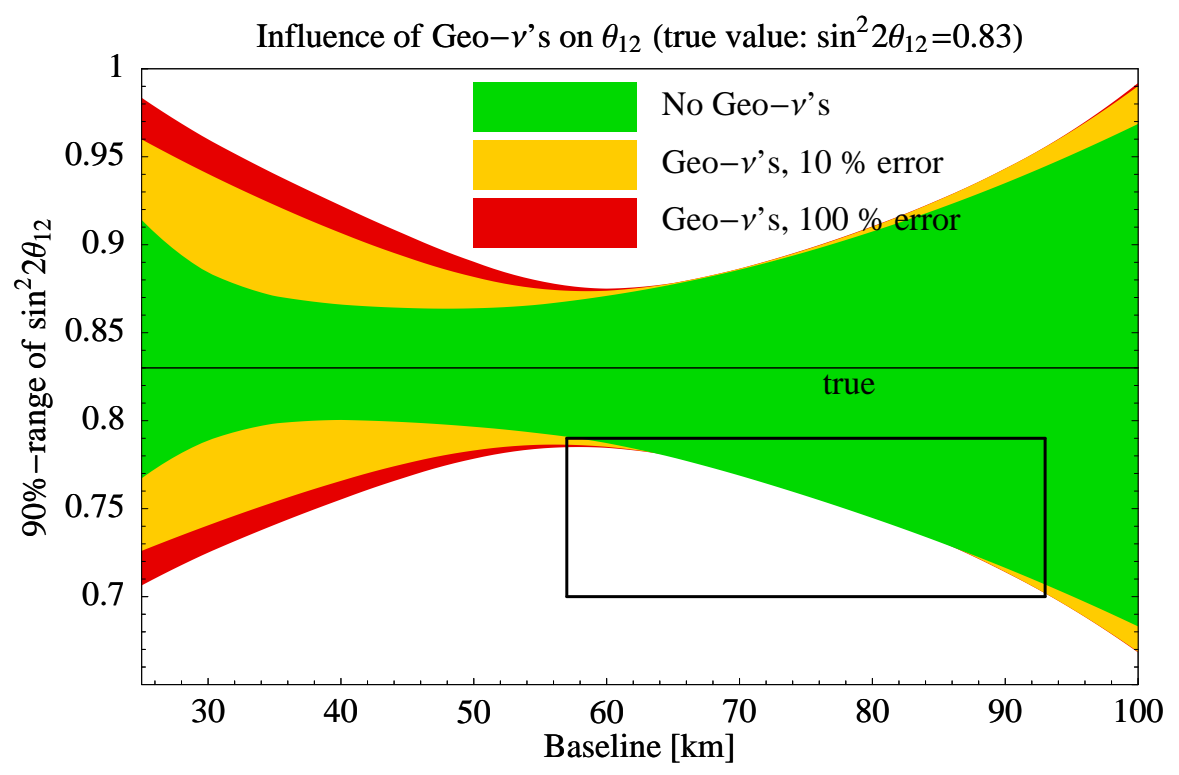

Figure 2: Sensitivity of LENA to $\theta_{12}$ for the assumed true value as a function of the baseline where the considered reactor has a thermal power of $0.5 \mathrm{GW}_{\mathrm{th}}$. The marked part is the region where the background self-calibration comes in. 


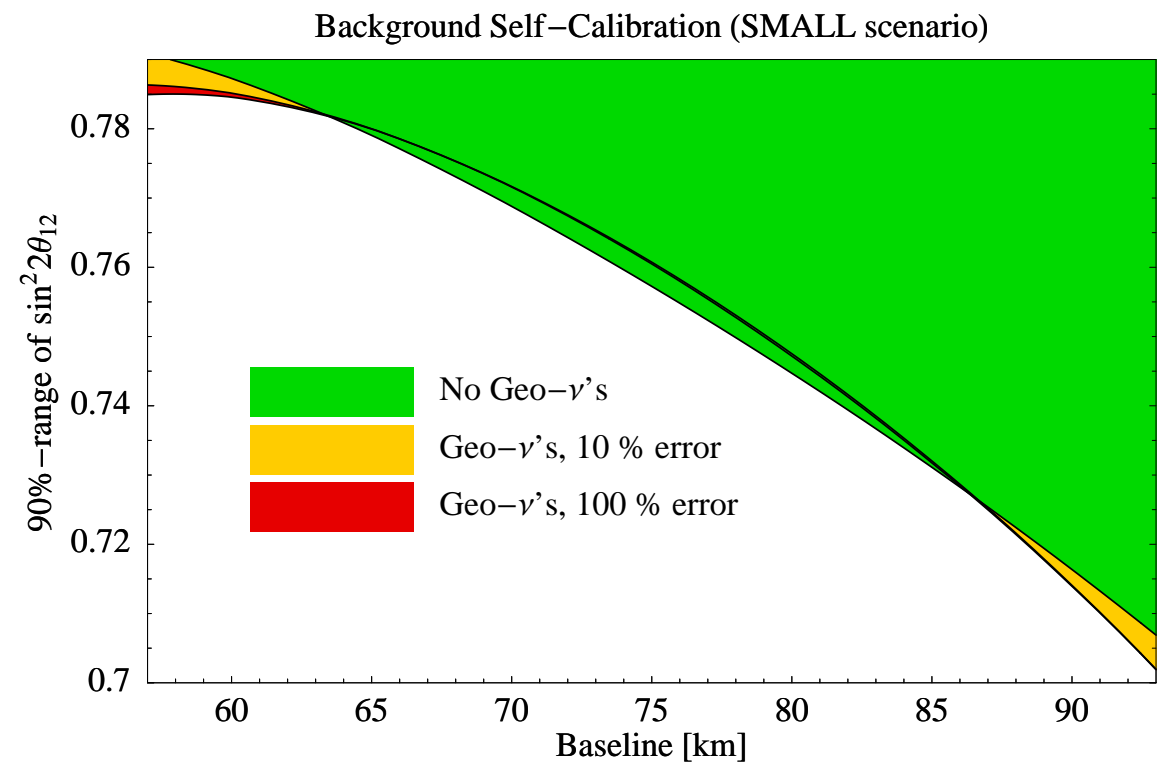

Figure 3: The rectangle from Fig. 2 drawn to a larger scale. Here, one can clearly see the seemingly paradoxial situation that a measurement with backgrounds can yield better results than one without.
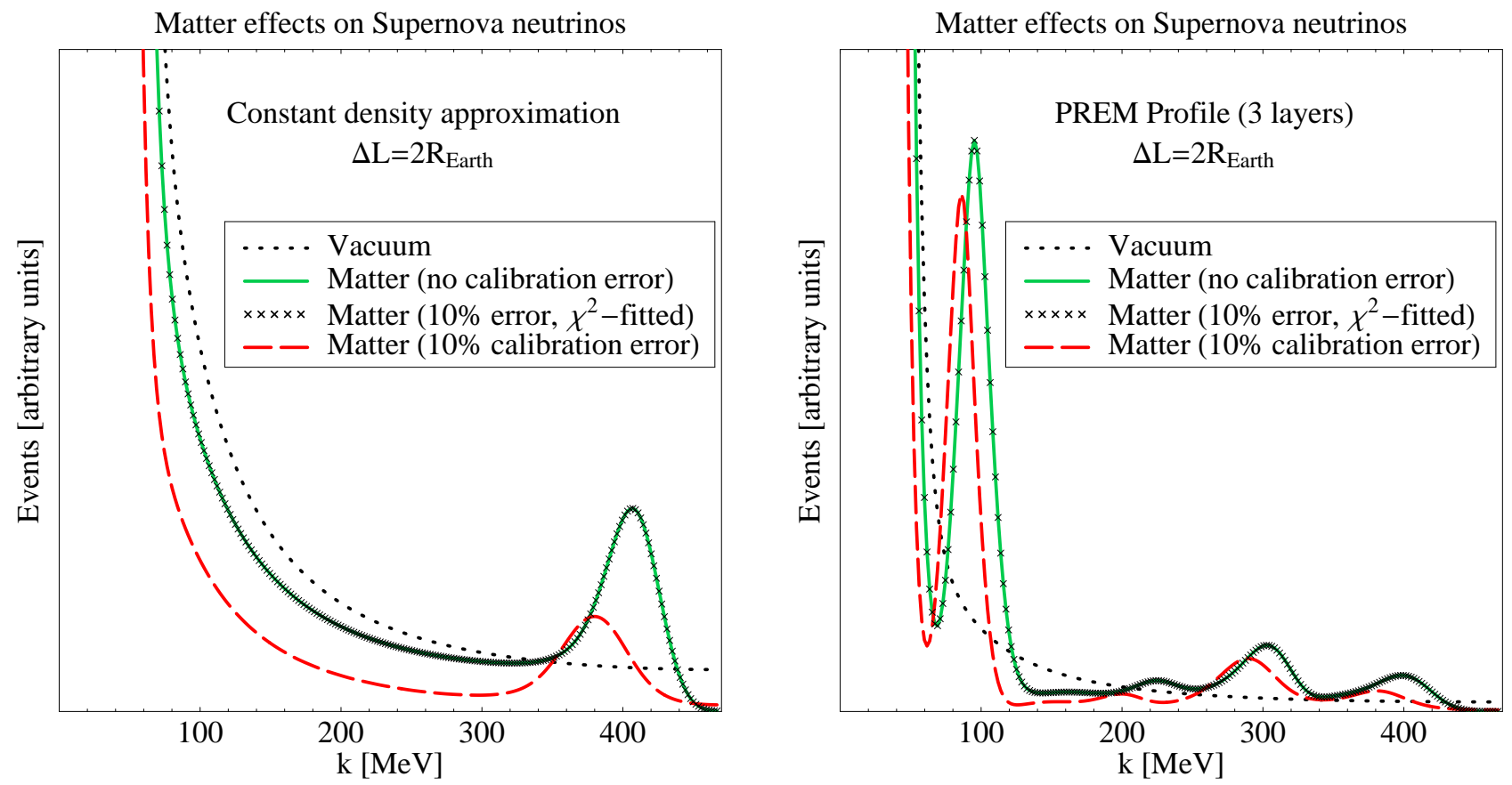

Figure 4: Matter effects on the power spectrum of supernova neutrinos for a constant density profile and a 3-layer approximation. The neutrinos always propagate through the whole diameter of the Earth. Considered are the cases of propagation through vacuum and through matter, while the latter is subdivided in the case with perfect energy calibration and the one with a $10 \%$ calibration error. Furthermore, for the wrong calibration, we have in one case performed a $\chi^{2}$ analysis and shifted the events with the fittet value of the energy calibration. Due to the self-calibration effect, this analysis has again given the correct adjustment of the energy, which causes the corrected data points to lie exactly on the line of perfect energy calibration. 